\title{
Hysteresis in linearly polarized nonresonantly driven exciton-polariton condensates
}

\author{
H. Sigurdsson $\odot^{*}$ \\ School of Physics and Astronomy, University of Southampton, Southampton SO17 1BJ, United Kingdom
}

(Received 25 March 2020; revised manuscript received 22 May 2020; accepted 27 May 2020; published 11 June 2020)

\begin{abstract}
It is shown that a condensate of microcavity exciton-polaritons exhibits bistability between its two orthogonal linearly polarized components for varying nonresonant excitation power. A non-Hermitian splitting of these components results in two parity-symmetric fixed-point solutions of opposite polarization, making up the hysteresis branches. These solutions correspond to the lowest threshold linearly polarized mode, which dominates at low pump powers, and a cross-polarized mode, which appears due to nonlinear pinning at higher excitation powers. The spin symmetry of the solutions extends the nonresonant bistable operation of polariton condensates to the entire equatorial plane of the Poincaré sphere. The results pave the way towards development of tailored bistable, linearly polarized, coherent sources of light.
\end{abstract}

DOI: 10.1103/PhysRevResearch.2.023323

\section{INTRODUCTION}

Semiconductor microcavity exciton-polaritons (here on polaritons) are spinor bosonic quasiparticles arising from the hybridization of microcavity embedded quantum well excitons and cavity photons in the strong light-matter coupling regime [1]. These particles possess a number of desirable properties including a very high Kerr-like nonlinearity due to their excitonic component and very fast, picosecond scale, dynamical response times from their photonic component. Moreover, under applied excitation, they can form a nonequilibrium counterpart of Bose-Einstein condensation where they amalgamate into a coherent macroscopic wave function with strong degree of polarization [2]. The polariton doublet spin structure is explicitly related to the polarization of the cavity photons through angular momentum selection rules [3]. This enables direct write and readout of polariton spin-information using polarized optical excitation elements, making them a promising platform to investigate driven-dissipative spin phenomena under controllable conditions. With the realization polariton condensation in now various materials at both cryogenic [2,4,5] and ambient conditions [6-8] (for review see Ref. [9]) their role for novel spinoptronic devices and application has enhanced [10,11].

Today, polariton condensates are conventionally realized under transverse confinement in the cavity plane where they occupy a well-defined trap ground state. This greatly simplifies the analysis of the condensate's complicated dynamics but still possesses a number of interesting features such as

\footnotetext{
*h.sigurdsson@soton.ac.uk

Published by the American Physical Society under the terms of the Creative Commons Attribution 4.0 International license. Further distribution of this work must maintain attribution to the author(s) and the published article's title, journal citation, and DOI.
}

weak lasing [12], limit cycles [13], chaos [14], symmetrybreaking bifurcations [15], and hysteresis [16,17]. Trapping of polariton condensates can today be realized through various methods such as the sample disorder [2], etched micropillars [18,19], optical trapping [20,21], and many more [22]. For resonantly excited polariton condensates, where the excitation laser is tuned in momentum and frequency with regards to the resonances of the polariton system, there has been an exciting development in understanding their bistable (or multistable) nature for the purpose of designing nonlinear devices such as spin switches [23-30]. There, switching occurs between different stable states due to particle interactions giving rise to a feedback effect between the intensity and the frequency of the polariton field which shifts it into resonance with the driving field.

However, for practical purposes it is desirable to extend these operational capabilities under nonresonant excitation schemes. Here, the beam's frequency is tuned just above the microcavity stop band which results in excitation of charge carriers which relax to form an incoherent ensemble of polaritons. At high-enough excitation densities the incoherent polaritons undergo stimulated bosonic scattering into a macroscopic coherent state which adopts a high degree of polarization [2,31]. The condensate in this nonresonantly driven-dissipative regime can possess multible stable solutions depending on its general complexity such as connectivity to other condensates [12,32], spatial [33,34], and spin structure $[16,17,35]$. However, nonresonant bistability in polariton condensates has only been investigated experimentally in the circular polarization degree of the cavity emission $[16,17,35]$ (corresponding to the two-level spin structure of the polariton field) with little focus on the degree of linear polarization in the condensate.

In this article, I demonstrate and analyze a bistable regime between two cross-linearly polarized components in a nonresonantly driven polariton condensate. The bistability belongs to two parity symmetry preserving solutions of the condensate. 
One solution corresponds to the lowest threshold condensation mode (dominant at low excitation powers) whereas the second mode belongs to a pinned cross-polarized state due to polariton-polariton interactions [36] (dominant at high pump power). The stability of the two solutions is confirmed through linear stability analysis on the driven-dissipative Gross-Pitaevskii equation, and numerically analyzed for elliptically polarized excitation beams. The parity symmetry of the two solutions generalizes the results to the entire equatorial plane of the Poincaré sphere, thus extending the application of polariton condensates in bistable spinoptronic devices.

\section{THEORY}

The spinor polariton condensate order parameter is written $\Psi=\left(\psi_{+}, \psi_{-}\right)^{T}$ where the two \pm 1 spin projections are defined along the [001] cavity growth axis (normal to the quantum well plane) and correspond explicitly to the $\sigma^{ \pm}$circular polarizations of the cavity light. The order parameter is modeled through a set of driven-dissipative Gross-Pitaevskii equations coupled to spin-polarized rate equations describing excitonic reservoirs $X_{\sigma}$ feeding the condensates [37]

$$
\begin{aligned}
i \frac{d \psi_{\sigma}}{d t} & =\frac{1}{2}\left[\alpha\left|\psi_{\sigma}\right|^{2}+g X_{\sigma}+i\left(R X_{\sigma}-\Gamma\right)\right] \psi_{\sigma}-\frac{\epsilon+i \gamma}{2} \psi_{-\sigma}, \\
\frac{d X_{\sigma}}{d t} & =-\left(\Gamma_{X}+R\left|\psi_{\sigma}\right|^{2}\right) X_{\sigma}+\Gamma_{s}\left(X_{-\sigma}-X_{\sigma}\right)+P_{\sigma}
\end{aligned}
$$

where $\sigma \in \pm$. Such a mean-field model has been applied successfully on a series of spinor polariton condensate experiments $[15,17,35]$ including recent reports of polarization pinning [38], and is the inspiration to the current study. Here, $\alpha$ and $g$ denote the polariton-polariton interaction and polariton-reservoir interaction, respectively, $R$ is the rate of stimulated scattering of polaritons into the condensate, and $\Gamma$ is the polariton decay rate. The spins are coupled by a nonHermitian coefficient $\epsilon+i \gamma$ representing splitting in both frequency $(\epsilon)$ and linewidth $(\gamma)$ between the linearly polarized $X Y$ components of the polaritons (i.e., $\left|\psi_{x, y}\right\rangle=\left[\left|\psi_{+}\right\rangle \pm\right.$ $\left.\left|\psi_{-}\right\rangle\right] / \sqrt{2}$ ). The former can appear due to the random sample birefringence [15,31,39], by applying strain which breaks the lateral symmetry [40], or by engineering confining potentials of reduced symmetry $[41,42]$. The latter appears from the finite curvature of the cavity stop band which translates to energy split $X$ and $Y$ modes experiencing different reflectivity from the cavity mirrors [15]. The parameter $\Gamma_{X}$ describes the decay rate of reservoir excitons, $\Gamma_{s}$ captures the spin relaxation rate between them, and $P_{ \pm}=P_{0} \cos ^{2}(\theta \mp \pi / 4)$ is the power of the nonresonant continuous wave pump where $\theta=0$ corresponds to zero circular polarization (i.e., cavity excited by a linearly polarized excitation), and $\theta= \pm 45^{\circ}$ corresponds to fully left- and right-clockwise circularly polarized light respectively. We point out that interactions between oppositespin polaritons are generally quite weak [43] compared to same-spin interactions $(\alpha)$ and therefore are neglected in this study.

The spin physics of the polaritons can be conveniently described in the pseudospin formalism [3] stemming from the fact that the measurable polarization of the emitted cavity light relates explicitly to the polariton spin structure. In what follows, we will not distinguish between the Stokes vector of the emitted light and the condensate pseudospin defined

$$
\mathbf{S}=\frac{1}{2} \Psi^{\dagger} \boldsymbol{\sigma} \Psi
$$

where $\sigma=\left(\hat{\sigma}_{x}, \hat{\sigma}_{y}, \hat{\sigma}_{z}\right)$ is the Pauli matrix vector. The components of the pseudospin $\mathbf{S}=\left(S_{x}, S_{y}, S_{z}\right)^{T}$, which are often denoted $\left(S_{1}, S_{2}, S_{3}\right)$, respectively, in the Stokes picture, are then written $S_{x}=\operatorname{Re}\left(\psi_{-}^{*} \psi_{+}\right), S_{y}=-\operatorname{Im}\left(\psi_{-}^{*} \psi_{+}\right)$, and $S_{z}=\left(\left|\psi_{+}\right|^{2}-\left|\psi_{-}\right|^{2}\right) / 2$. The total pseudospin is defined $S=$ $\sqrt{S_{x}^{2}+S_{y}^{2}+S_{z}^{2}}=\left(\left|\psi_{+}\right|^{2}+\left|\psi_{-}\right|^{2}\right) / 2$. The pseudospin components equations of motion following from Eq. (1a) are written

$$
\begin{gathered}
\frac{d S_{x}}{d t}=f_{1}=-G S_{y}+W S_{x}-\gamma S-\alpha S_{z} S_{y}, \\
\frac{d S_{y}}{d t}=f_{2}=G S_{x}+W S_{y}+\epsilon S_{z}+\alpha S_{z} S_{x}, \\
\frac{d S_{z}}{d t}=f_{3}=W^{\prime} S+W S_{z}-\epsilon S_{y}, \\
\frac{d S}{d t}=W^{\prime} S_{z}+W S-\gamma S_{x},
\end{gathered}
$$

where $G=g\left(X_{+}-X_{-}\right) / 2, \quad W=R\left(X_{+}+X_{-}\right) / 2-\Gamma$, and $W^{\prime}=R\left(X_{+}-X_{-}\right) / 2$. The splitting $\epsilon$ can be regarded as an effective in-plane magnetic field $\boldsymbol{\Omega}_{\|}=\left(\Omega_{x}, \Omega_{y}\right)=\epsilon \hat{\mathbf{x}}$ rotating the polariton pseudospin $\dot{\mathbf{S}} \propto \mathbf{S} \times \boldsymbol{\Omega}_{\|}$, where $\hat{\mathbf{x}} \|[110]$ and $\hat{\mathbf{y}} \|[1 \overline{1} 0]$ are the coordinate unit vectors of the cavity plane. In terms of the pseudospin components, Eq. (1b) can be simply written

$$
\frac{d X_{ \pm}}{d t}=f_{4,5}=-\left[\Gamma_{X}+R\left(S \pm S_{z}\right)\right] X_{ \pm}+\Gamma_{s}\left(X_{\mp}-X_{ \pm}\right)+P_{ \pm} \text {. }
$$

The general stationary solution to Eqs. (3) to (7) is cumbersome and we will only focus on two stationary solutions which are of interest to this study. These two solutions, under the condition of linearly polarized excitation $P=P_{ \pm}>0$, correspond to a balanced spin population of the excitonic reservoirs $X=X_{ \pm}$and therefore preserve the spin symmetry of the system. Substitution of these conditions into the equations of motion gives these two stationary solutions as

$$
\begin{aligned}
& S_{x}= \pm S, \quad S_{y}=0, \quad S_{z}=0, \\
& S=\frac{P}{\Gamma \pm \gamma}-\frac{\Gamma_{X}}{R}, \quad X=\frac{\Gamma \pm \gamma}{R} .
\end{aligned}
$$

These two solutions represent a polariton condensate emitting $X$ and $Y$ polarized light, respectively. We note that the regime where $S<0$ is unphysical as a negative number of polaritons cannot exist in the condensate. It then defines the excitation threshold of the system

$$
P_{\mathrm{th}}=\frac{\Gamma_{X}(\Gamma-\gamma)}{R} .
$$

If $P<P_{\text {th }}$ then the only stable solution of the system is $S=0$. If $P>P_{\text {th }}$ then the system quickly departs from $S=0$ and assumes the condition of Eq. (9), which corresponds to the $S_{x}=-S$ solution. This is the so-called first lasing state or the condensate solution with the lowest threshold (the lowest 

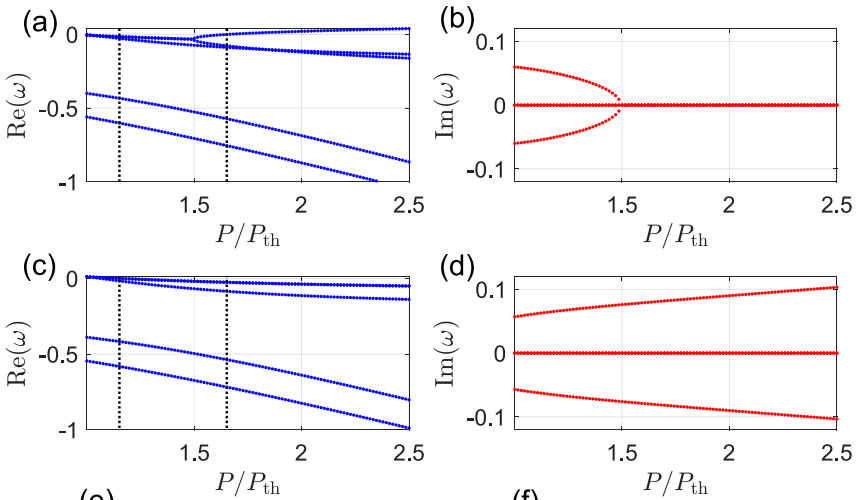

(e)
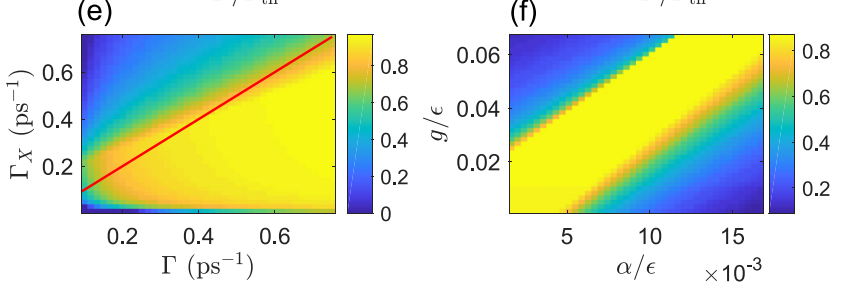

FIG. 1. (a), (c) Real and (b), (d) imaginary parts of the eigenvalues $\omega$ for $\mathbf{u}_{X, Y}$, respectively, as a function of excitation power. The vertical dotted lines indicate a region where all $\operatorname{Re}(\omega)$ between (a) and (c) are negative, marking that both stationary solutions $\mathbf{u}_{X, Y}$ are stable. (e), (f) Existence of bistability $\mathcal{B}$ plotted (heatmap) as a function of $\left(\Gamma_{X}, \Gamma\right)$ and $(g, \alpha)$, respectively. Yellow color denotes greater bistability and red line denotes $\Gamma=\Gamma_{x}$.

amount of power needed to stimulate the system and trigger condensation). The second solution $S_{x}=S$ corresponds to polarization pinning. As the particle number in the condensate increases with the pump power the polariton-polariton interactions start pinning the pseudospin along the in-plane magnetic field $\boldsymbol{\Omega}[36]$.

Although $S_{x}=-S$ might have the lowest threshold we have not verified whether it is stable. Indeed, as soon as one reaches $P=P_{\text {th }}$ one might depart immediately from $S_{x}=-S$ after the initial transients of the system into a different fixed point, a limit cycle, or a chaotic trajectory [14] depending on the parameters of Eqs. (3) to (7). To verify the stability of the condensate stationary solutions, which we denote $\mathbf{u}=\left(S_{x}, S_{y}, S_{z}, X_{+}, X_{-}\right)^{T}$, one needs to construct the Jacobian matrix $\mathbf{J}$ corresponding to the pseudospin equation of motion and analyze its eigenvalues $\omega$ (Lyapunov exponents)

$$
\mathbf{J}(\mathbf{u}) \mathbf{v}=\omega \mathbf{v} .
$$

The elements of the Jacobian are written

$$
J_{n m}=\frac{\partial f_{n}(\mathbf{u})}{\partial x_{m}},
$$

where $x_{1,2,3}=S_{x, y, z}$ and $x_{4,5}=X_{ \pm}$. According to the linear stability theorem, the eigenvalues $\omega$ determine the evolution of perturbations around the equilibrium points (stationary solutions) $\mathbf{u}$. $\operatorname{Im}(\omega)$ contributes to an oscillatory part of the perturbation, whereas $\operatorname{Re}(\omega)$ dictates whether the point $\mathbf{u}$ is stable $[\operatorname{Re}(\omega)<0]$ or unstable $[\operatorname{Re}(\omega)>0]$. In Fig. 1 we plot the eigenvalues $\omega$ for the two solutions of interest corresponding to a $X$ and $Y$ polarized condensate

$$
\mathbf{u}_{X, Y}=\left(\begin{array}{c} 
\pm\left[\frac{P}{\Gamma \pm \gamma}-\frac{\Gamma_{X}}{R}\right] \\
0 \\
0 \\
\frac{\Gamma \pm \gamma}{R}, \\
\frac{\Gamma \pm \gamma}{R}
\end{array}\right) .
$$

Figures 1 (a) to 1 (d) show the real and imaginary $\omega$ for $\mathbf{u}_{X, Y}$. Interestingly, the two solutions share a region enclosed by the two vertically dotted lines where all real eigenvalues are negative. Therefore, the system is bistable in this region and can exhibit hysteresis. We stress that the choice of the in-plane magnetic field orientation $\boldsymbol{\Omega}_{\|}$is completely arbitrary as it does not break the parity symmetry between the spin-up and spin-down components. One can therefore design bistable condensates which operate between polarization angles $\phi=$ $\tan ^{-1}\left(\Omega_{y} / \Omega_{x}\right)$ and $\phi+\pi$ by simply adjusting the orientation of $\Omega_{\|}$. The parameters in all calculations are taken similar to those used in past experiments [15] and are found in Ref. [44].

It is instructive to check the effects of different parameters in Eq. (1) on the bistable range determined from the Lyapunov exponents $\operatorname{Re}(\omega)$. In Figs. 1(e) and 1(f) we calculate the "existence" of bistability defined as

$$
\mathcal{B}=\frac{\Delta}{P_{2}-P_{1}} .
$$

Here, $\Delta$ is the range of all Lyapunov exponents belonging to both $\mathbf{u}_{X, Y}$ having a negative real part going from pump power $P_{1} \rightarrow P_{2}$ which are taken $P_{1}=P_{\mathrm{th}}$ and $P_{2}=2 P_{\mathrm{th}}$, respectively. When $\mathcal{B}=1$ both $\mathbf{u}_{X, Y}$ are stable over the whole investigated pump range whereas $\mathcal{B}=0$ means that no common stability was shared between them. In Fig. 1(e) we observe that for reservoir decay rate lower than the polariton decay rate one recovers strong bistability (yellow color). The red line denotes $\Gamma_{X}=\Gamma$ and indicates that a more slowly decaying reservoir $\left(\Gamma_{x}<\Gamma\right)$ is favorable for the bistability. In Fig. 1(f) we observe a more stringent regime of bistability between $g$ and $\alpha$ which can be intuitively understood from the following consideration. If $\alpha$ is strong then the condensate is more preferably pinned and assumes the $\mathbf{u}_{X}$ solution quickly after threshold resulting in a smaller bistable range (blue-ish lower right corner). On the other hand, if $g$ is strong then the reservoir weakens the pinned solution $\mathbf{u}_{X}$ through an effective focusing nonlinearity which makes the $\mathbf{u}_{Y}$ state dominant and reduces the bistable range (blue-ish upper left corner). This focusing nonlinearity becomes apparent by considering $\Gamma_{s}=0$ and expanding the steady state of the reservoir

$$
X_{\sigma}=\frac{P_{\sigma}}{\Gamma_{X}+R\left|\psi_{\sigma}\right|^{2}} \simeq \frac{P_{\sigma}}{\Gamma_{X}}\left[1-\frac{R\left|\psi_{\sigma}\right|^{2}}{\Gamma_{X}}+\mathcal{O}\left(\left|\psi_{\sigma}\right|^{4}\right)\right] .
$$

For optically trapped condensates where the reservoir is separated from the condensate $g$ is generally smaller than $\alpha$ and one can retrieve the bistability by simply adjusting $\alpha$ through the condensate confinement area $\alpha \propto \int|\Psi(\mathbf{r})|^{4} d \mathbf{r}$. Moreover, it holds that $\alpha / g \propto|\chi|^{2}$ where $|\chi|^{2}$ is the excitonic Hopfield fraction of the polariton [1], which can then also be adjusted to obtain bistable operation. We point out that for Figs. 1(a) to 

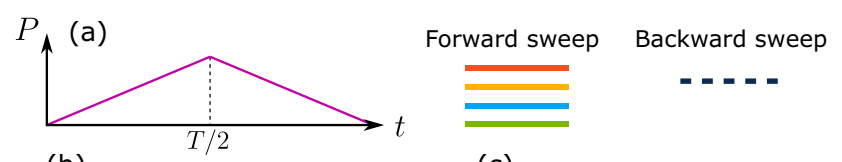

(b)

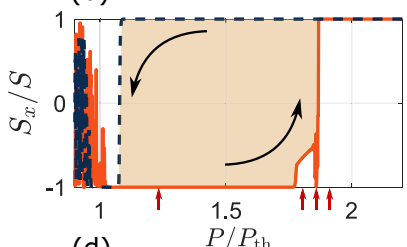

(c)
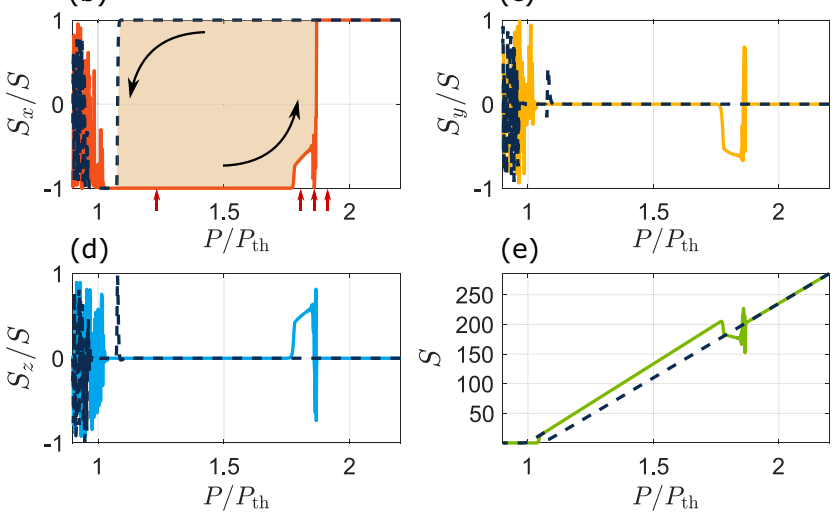

(e)

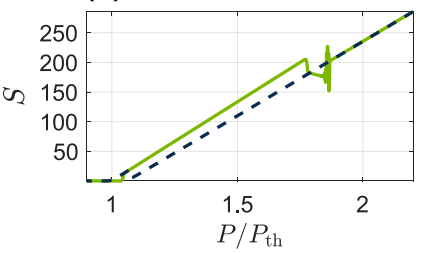

FIG. 2. (a) Schematic of the triangular nonresonant pulse. (b)-(e) Instantaneously extracted values of the normalized pseudospin components $S_{x, y, z} / S$ and $S$ for different values of pump power. Colored whole lines denote a forward/increasing sweep in power and dark dashed lines denote a backward/decreasing sweep in power. The orange shaded region denotes the hysteresis and black arrows the path of the pseudospin. Small red arrows in (b) indicate the states shown in Fig. 3.

1(d) and 1(f) the parameters used [44] give particle numbers $S \approx 120$ and $X_{\sigma} \approx 130$ at $P=1.5 P_{\text {th }}$.

The bistable nature of the system is verified by numerically integrating Eq. (1) and sweeping the pump power $P$ adiabatically [i.e., much more slowly than the characteristic timescales of Eq. (1)] from low to high power and back again. In an experiment, this corresponds to a long triangular-shaped excitation pulse of duration $T$. Additionally, we introduce a stochastic Gaussian white noise term $\eta_{\sigma}(t)$ to the right-hand side of Eq. (1a), which introduces weak perturbations the condensate at each timestep. Physically, such a stochastic term $[36,45]$ mimics the shot noise in the system and serves to test the stability of the pseudospin at its equilibrium points. The noise is defined through its correlators $\left\langle d \eta_{\sigma}(t) d \eta_{\sigma^{\prime}}\left(t^{\prime}\right)\right\rangle \propto$ $\delta_{\sigma \sigma^{\prime}} \delta\left(t-t^{\prime}\right)$ and $\left\langle d \eta_{\sigma}(t) d \eta_{\sigma^{\prime}}^{*}\left(t^{\prime}\right)\right\rangle=0$. Results are shown in Fig. 2 where the forward sweep is denoted by colored whole lines and backward sweep with dark dashed lines. In the forward sweep four distinct regimes denoted by the small red arrows can be observed [see Fig. 2(b)]. The polarization first adopts $S_{x}=-S$ state after threshold $P_{\text {th }}$. The state stays stable until it destabilizes corresponding to the second dotted line in Fig. 1(a). The pseudospin then bifurcates into a new stationary state (second red arrow from left) with net circular and diagonal polarization $\left(P / P_{\text {th }} \approx 1.75\right)[14,15]$, which, depending on the initial dynamics, is either orientated $S_{y}>0$ and $S_{z}<0$, or $S_{y}<0$ and $S_{z}>0$ [see Figs. 2(c) and 2(d) for the latter case]. As such, the symmetry of the system is broken spontaneously similar to macroscopic quantum self trapping in bosonic Josephson junctions [46-48]. At higher powers the pseudospin departs from the bifurcated solution into a limit cycle $\left(P / P_{\text {th }} \approx 1.85\right)$ denoted by the third red arrow from the left. Such limit cycle states have been reported experimentally in coupled polariton condensates [49] and correspond to oscil-
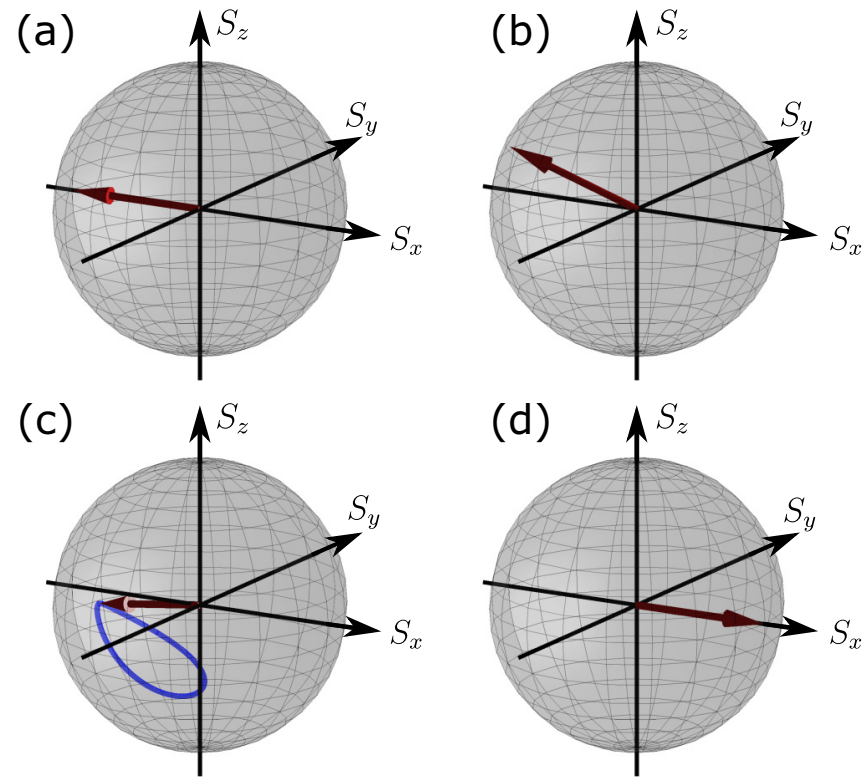

FIG. 3. (a)-(d) Normalized pseudospin states (dark red arrows) shown on the Poincaré sphere at $P / P_{\mathrm{th}}=1.2,1.75,1.85,1.9$, respectively, corresponding to (from left to right) the small red arrows in Fig. 2(b). In panel (c) the closed blue trajectory denotes the cyclical path which the pseudospin follows in time.

latory behavior in the condensate pseudospin components and reservoirs. Lastly, the condensate departs from the limit cycle into the upper bistable branch given by the pinned solution $S_{x}=S\left(P / P_{\mathrm{th}} \approx 1.9\right)$ denoted by the fourth red arrow from the left. In Figs. 3(a) to 3(d) we show the four above-mentioned pseudospin states on the surface of the normalized Poincaré sphere corresponding to the four small red arrows in Fig. 2(b). The blue curve in Fig. 3(c) traces the trajectory of the limit cycle state on the sphere's surface with the dark red arrow representing the pseudospin in a single instance of time.

The dark dashed lines in Fig. 2 denote the pseudospin state as the power is swept back (decreased). As suggested by the stability analysis presented in Fig. 1, there exists a large hysteresis in the $S_{x}$ component with the $S_{x}=S$ state extending all the way back to the leftmost vertically dotted line in Figs. 1(a) and 1(c). The collapse of the $S_{x}=S$ state is accompanied with sharp transients in the $S_{y}$ and $S_{z}$ components. We note that in Fig. 2(e) the two linear-like trajectories of the total spin population $S$ corresponding to $\mathbf{u}_{X, Y}$ are shifted with respect to one another because of the linewidth difference $\gamma$ [see Eq. (8)].

We next move onto the condition of $P_{-} \neq P_{+}$and analyze the expanse of the hysteresis for excitation schemes with varying incident polarization $\theta$. This gives a sense of robustness of the hysteresis regime against the excitation induced out-of-plane ( $\hat{\mathbf{z}} \|$ [001]) magnetic field written

$$
\boldsymbol{\Omega}_{\perp}=\Omega_{z} \hat{\mathbf{z}}=\left(\alpha S_{z}+G\right) \hat{\mathbf{z}} .
$$

Physically, such a field arises due to the different blueshift experienced by the spin-up and spin-down condensate polaritons due to interactions between themselves and the background reservoir, which causes an effective Zeeman splitting [50]. It 

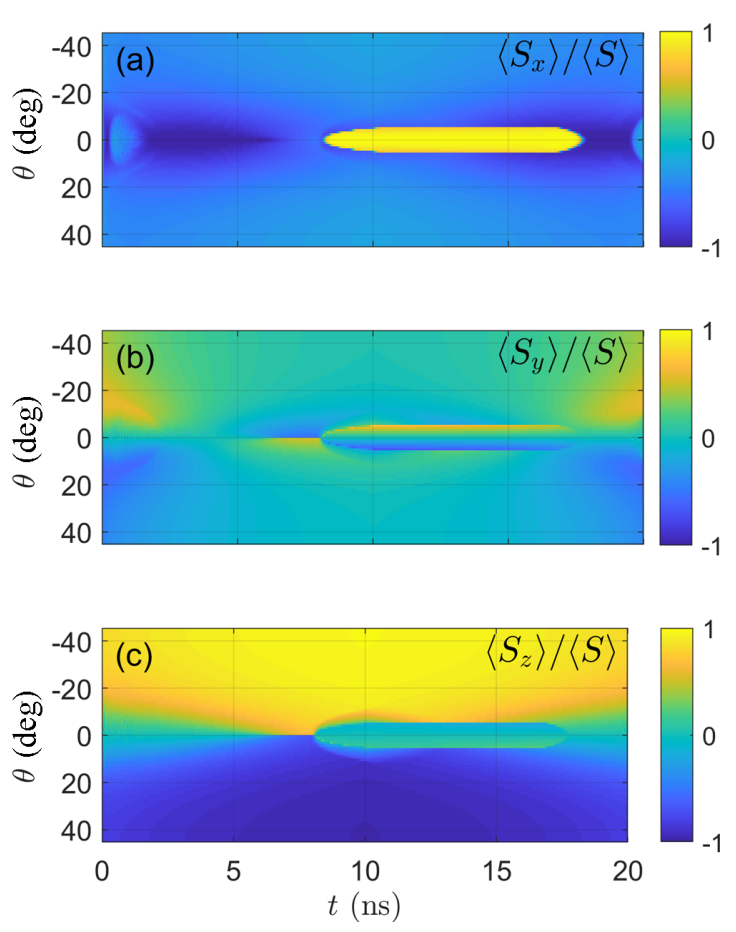

FIG. 4. (a)-(c) Normalized average pseudospin states for a triangular pulse with $T=20 \mathrm{~ns}$ shown in Fig. 2(a). The starting and peak pump values are $P_{0}(t=0) / P_{\text {th }}=0.8$ and $P_{0}(t=T / 2) / P_{\text {th }}=2.3$. The average is performed over $N=200$ trials.

can therefore be expected that the hysteresis in Fig. 2(a) will diminish as the net effective magnetic field $\boldsymbol{\Omega}=\boldsymbol{\Omega}_{\|}+\boldsymbol{\Omega}_{\perp}$ starts tilting more and more in the $\hat{\mathbf{z}}$ direction. Moreover, the spin relaxation parameter $\Gamma_{s}$ will now play a role in balancing the populations of the reservoirs against the elliptically polarized excitation. To investigate these effects, we plot in Fig. 4 heatmaps showing the pulse-averaged pseudospin components for varying $\theta$. The pulse is triangular shaped [see Fig. 2(a)] with a duration $T=20 \mathrm{~ns}$. The system is bistable if, for a given $\theta$, two opposite equidistant points from the line $t=T / 2$ are differently valued. For example, looking at Fig. 4(a), the $S_{x}$ polarization at $t=5 \mathrm{~ns}$ and $t=15 \mathrm{~ns}$ for $\theta=0$ are opposite, corresponding to the hysteresis in Fig. 2(b). The pulse average is written $\left\langle S_{j}\right\rangle=\frac{1}{N} \sum_{n} S_{j}$ where $N$ denotes the number of pulses, each with a random initial condition. The results in Fig. 4(a) show that the bistable range vanishes when the ellipticity $|\theta|$ of the pump increases, resulting more and more in a condensate which is pinned along the interaction induced magnetic field $\boldsymbol{\Omega}_{\perp}$ [yellow and blue regimes in Fig. 4(c)]. The diagonal component in Fig. 4(b), for low $|\theta|$, becomes polarized when the system adopts the spinbifurcated state [Fig. 3(b)] which reverses when the pinned state takes over [Fig. 3(d)]. The extent of the bistable regime is around $\theta \approx \pm 6^{\circ}$ which demonstrates that its presence is not jeopardized in the case of slight ellipticity in the incident optical excitation.

It is worth noting that energy relaxation of polaritons can affect the bistability observed. This can be understood from the fact that a damping mechanism competes with the linewidth difference $\gamma$ and therefore can cause polaritons to immediately condense into the $X$ polarized ground state and the bistability to be lost. Such damping, taken to be proportional to the gain of the condensate [51], can be introduced to Eq. (1a) such that it reads

$$
\begin{aligned}
i \frac{d \psi_{\sigma}}{d t}= & \frac{1}{2}\left[\alpha\left|\psi_{\sigma}\right|^{2}+g X_{\sigma}+i\left(R X_{\sigma}-\Gamma\right)+\frac{R X_{\sigma}}{\Omega} \frac{d}{d t}\right] \psi_{\sigma} \\
& -\frac{\epsilon+i \gamma}{2} \psi_{-\sigma}
\end{aligned}
$$

where $\Omega^{-1}$ denotes the strength of the damping. The above equation possesses a new threshold condition for the two solutions $S_{x}= \pm S$ which allow one to derive an approximate analytical inequality preserving the bistability of the system

$$
\Omega>\frac{\Gamma \epsilon}{2 \gamma}, \quad \text { Bistable system. }
$$

Equation (17) can be understood as follows. For increasing $\epsilon$ the energy splitting of the two bistable modes introduces a greater difference in the lifetime of the modes due to damping, leading eventually to the condensation of polaritons into the $X$ polarized ground state. For large $\gamma$ the difference in the mode linewidths due to different reflectance of the cavity mirrors is strong and overcomes any damping effects. From past experiments $[15,17,35]$ the ratio is typically $\epsilon / \gamma \approx 5$, which is much lower than the value of $\Omega$ used in a recent study [51]. We also note that $\Gamma$ enters Eq. (17) which allows one to retrieve the bistable regime by either changing the quality of the cavity or the detuning between the cavity photon and exciton resonances.

\section{CONCLUSION}

I theoretically demonstrated and analyzed a parity (spin) symmetry preserving hysteresis in a system of trapped driven-dissipative spinor exciton polariton condensates for varying nonresonant excitation powers. This effect is realized through splitting of the two orthogonal linearly polarized modes which also, importantly, experience different linewidths. The hysteresis is then found to be dominantly made up of two spin symmetric solutions $S_{x}= \pm S$, where the former corresponds to the lowest threshold mode (dominant at low excitation power), and the latter appears due to pinning along an in-plane magnetic field $\boldsymbol{\Omega}_{\|}$(dominant at high excitation power). Due to the symmetric structure of the equations of motion, the hysteresis is realizable on the entire equatorial plane of the Poincaré sphere through control of $\boldsymbol{\Omega}_{\|}$, thus improving the applicability of polariton condensates as coherent bistable sources of linearly polarized light.

\section{ACKNOWLEDGMENT}

The author acknowledges the support of the UK's Engineering and Physical Sciences Research Council (Grant No. EP/M025330/1 on Hybrid Polaritonics). 
[1] A. Kavokin, J. J. Baumberg, G. Malpuech, and F. P. Laussy, Microcavities (Oxford University Press, Oxford, 2007).

[2] J. Kasprzak, M. Richard, S. Kundermann, A. Baas, P. Jeambrun, J. M. J. Keeling, F. M. Marchetti, M. H. Szymańska, R. André, J. L. Staehli, V. Savona, P. B. Littlewood, B. Deveaud, and L. S. Dang, Nature 443, 409 (2006).

[3] K. V. Kavokin, I. A. Shelykh, A. V. Kavokin, G. Malpuech, and P. Bigenwald, Phys. Rev. Lett. 92, 017401 (2004).

[4] R. Balili, V. Hartwell, D. Snoke, L. Pfeiffer, and K. West, Science 316, 1007 (2007).

[5] M. Waldherr, N. Lundt, M. Klaas, S. Betzold, M. Wurdack, V. Baumann, E. Estrecho, A. Nalitov, E. Cherotchenko, H. Cai, E. A. Ostrovskaya, A. V. Kavokin, S. Tongay, S. Klembt, S. Höfling, and C. Schneider, Nat. Commun. 9, 3286 (2018).

[6] S. Kéna-Cohen and S. R. Forrest, Nat. Photon. 4, 371 (2010).

[7] J. D. Plumhof, T. Stöferle, L. Mai, U. Scherf, and R. F. Mahrt, Nat. Mater. 13, 247 (2014).

[8] R. Su, S. Ghosh, J. Wang, S. Liu, C. Diederichs, T. C. H. Liew, and Q. Xiong, Nat. Phys. 16, 301 (2020).

[9] T. Guillet and C. Brimont, C. R. Phys. 17, 946 (2016).

[10] T. Liew, I. Shelykh, and G. Malpuech, Physica E (Amsterdam) 43, 1543 (2011).

[11] D. Sanvitto and S. Kéna-Cohen, Nat. Mater. 15, 1061 (2016).

[12] I. L. Aleiner, B. L. Altshuler, and Y. G. Rubo, Phys. Rev. B 85, 121301(R) (2012).

[13] K. Rayanov, B. L. Altshuler, Y. G. Rubo, and S. Flach, Phys. Rev. Lett. 114, 193901 (2015).

[14] R. Ruiz-Sánchez, R. Rechtman, and Y. G. Rubo, Phys. Rev. B 101, 155305 (2020)

[15] H. Ohadi, A. Dreismann, Y. G. Rubo, F. Pinsker, Y. del ValleInclan Redondo, S. I. Tsintzos, Z. Hatzopoulos, P. G. Savvidis, and J. J. Baumberg, Phys. Rev. X 5, 031002 (2015).

[16] L. Pickup, K. Kalinin, A. Askitopoulos, Z. Hatzopoulos, P. G. Savvidis, N. G. Berloff, and P. G. Lagoudakis, Phys. Rev. Lett. 120, 225301 (2018).

[17] Y. del Valle-Inclan Redondo, H. Sigurdsson, H. Ohadi, I. A. Shelykh, Y. G. Rubo, Z. Hatzopoulos, P. G. Savvidis, and J. J. Baumberg, Phys. Rev. B 99, 165311 (2019).

[18] D. Bajoni, P. Senellart, E. Wertz, I. Sagnes, A. Miard, A. Lemaître, and J. Bloch, Phys. Rev. Lett. 100, 047401 (2008).

[19] M. Galbiati, L. Ferrier, D. D. Solnyshkov, D. Tanese, E. Wertz, A. Amo, M. Abbarchi, P. Senellart, I. Sagnes, A. Lemaître, E. Galopin, G. Malpuech, and J. Bloch, Phys. Rev. Lett. 108, 126403 (2012).

[20] A. Askitopoulos, H. Ohadi, A. V. Kavokin, Z. Hatzopoulos, P. G. Savvidis, and P. G. Lagoudakis, Phys. Rev. B 88, 041308(R) (2013).

[21] P. Cristofolini, A. Dreismann, G. Christmann, G. Franchetti, N. G. Berloff, P. Tsotsis, Z. Hatzopoulos, P. G. Savvidis, and J. J. Baumberg, Phys. Rev. Lett. 110, 186403 (2013).

[22] C. Schneider, K. Winkler, M. D. Fraser, M. Kamp, Y. Yamamoto, E. A. Ostrovskaya, and S. Höfling, Rep. Prog. Phys. 80, 016503 (2016).

[23] N. A. Gippius, I. A. Shelykh, D. D. Solnyshkov, S. S. Gavrilov, Y. G. Rubo, A. V. Kavokin, S. G. Tikhodeev, and G. Malpuech, Phys. Rev. Lett. 98, 236401 (2007).
[24] A. Amo, T. C. H. Liew, C. Adrados, R. Houdré, E. Giacobino, A. V. Kavokin, and A. Bramati, Nat. Photon. 4, 361 (2010).

[25] D. Sarkar, S. S. Gavrilov, M. Sich, J. H. Quilter, R. A. Bradley, N. A. Gippius, K. Guda, V. D. Kulakovskii, M. S. Skolnick, and D. N. Krizhanovskii, Phys. Rev. Lett. 105, 216402 (2010).

[26] T. K. Paraïso, M. Wouters, Y. Léger, F. Morier-Genoud, and B. Deveaud-Plédran, Nat. Mater. 9, 655 (2010).

[27] D. V. Vishnevsky, D. D. Solnyshkov, N. A. Gippius, and G. Malpuech, Phys. Rev. B 85, 155328 (2012).

[28] O. Bozat, I. G. Savenko, and I. A. Shelykh, Phys. Rev. B 86 , 035413 (2012)

[29] M. Wouters, T. K. Paraïso, Y. Léger, R. Cerna, F. MorierGenoud, M. T. Portella-Oberli, and B. Deveaud-Plédran, Phys. Rev. B 87, 045303 (2013)

[30] R. Cerna, Y. Léger, T. K. Paraïso, M. Wouters, F. MorierGenoud, M. T. Portella-Oberli, and B. Deveaud, Nat. Commun. 4, 2008 (2013).

[31] J. Kasprzak, R. André, L. S. Dang, I. A. Shelykh, A. V. Kavokin, Y. G. Rubo, K. V. Kavokin, and G. Malpuech, Phys. Rev. B 75 , 045326 (2007).

[32] E. B. Magnusson, H. Flayac, G. Malpuech, and I. A. Shelykh, Phys. Rev. B 82, 195312 (2010).

[33] E. Z. Tan, H. Sigurdsson, and T. C. H. Liew, Phys. Rev. B 97, 075305 (2018).

[34] X. Ma and S. Schumacher, Phys. Rev. Lett. 121, 227404 (2018).

[35] A. Dreismann, H. Ohadi, Y. del Valle-Inclan Redondo, R. Balili, Y. G. Rubo, S. I. Tsintzos, G. Deligeorgis, Z. Hatzopoulos, P. G. Savvidis, and J. J. Baumberg, Nat. Mater. 15, 1074 (2016).

[36] D. Read, T. C. H. Liew, Y. G. Rubo, and A. V. Kavokin, Phys. Rev. B 80, 195309 (2009).

[37] M. Wouters and I. Carusotto, Phys. Rev. Lett. 99, 140402 (2007).

[38] I. Gnusov, H. Sigurdsson, S. Baryshev, T. Ermatov A. Askitopoulos, and P. G. Lagoudakis, arXiv:2004.11607.

[39] Ł. Kłopotowski, M. Martín, A. Amo, L. Viña, I. Shelykh, M. Glazov, G. Malpuech, A. Kavokin, and R. André, Solid State Commun. 139, 511 (2006).

[40] A. A. Demenev, Y. V. Grishina, A. V. Larionov, N. A. Gippius, C. Schneider, S. Höfling, and V. D. Kulakovskii, Phys. Rev. B 96, 155308 (2017).

[41] M. Klaas, O. A. Egorov, T. C. H. Liew, A. Nalitov, V. Marković, H. Suchomel, T. H. Harder, S. Betzold, E. A. Ostrovskaya, A. Kavokin, S. Klembt, S. Höfling, and C. Schneider, Phys. Rev. B 99, 115303 (2019).

[42] S. Gerhardt, M. Deppisch, S. Betzold, T. H. Harder, T. C. H Liew, A. Predojević, S. Höfling, and C. Schneider, Phys. Rev. B 100, 115305 (2019)

[43] C. Ciuti, V. Savona, C. Piermarocchi, A. Quattropani, and P. Schwendimann, Phys. Rev. B 58, 7926 (1998).

[44] $\Gamma=0.2 \mathrm{ps}^{-1}, \Gamma_{X}=2 \Gamma, \Gamma_{s}=0.2 \Gamma_{X}, \epsilon=0.06 \mathrm{ps}^{-1}, \gamma=0.2 \epsilon$, $\alpha=5 \times 10^{-4} \mathrm{ps}^{-1}, R=3 \alpha, g=\alpha, T=20 \mathrm{~ns}$.

[45] A. Chiocchetta and I. Carusotto, Europhys. Lett. 102, 67007 (2013)

[46] M. Albiez, R. Gati, J. Fölling, S. Hunsmann, M. Cristiani, and M. K. Oberthaler, Phys. Rev. Lett. 95, 010402 (2005).

[47] I. A. Shelykh, D. D. Solnyshkov, G. Pavlovic, and G. Malpuech, Phys. Rev. B 78, 041302(R) (2008). 
[48] M. Abbarchi, A. Amo, V. G. Sala, D. D. Solnyshkov, H. Flayac, L. Ferrier, I. Sagnes, E. Galopin, A. Lemaître, G. Malpuech, and J. Bloch, Nat. Phys. 9, 275 (2013).

[49] S. Kim, Y. G. Rubo, T. C. H. Liew, S. Brodbeck, C. Schneider, S. Höfling, and H. Deng, Phys. Rev. B 101, 085302 (2020).
[50] I. Shelykh, G. Malpuech, K. V. Kavokin, A. V. Kavokin, and P. Bigenwald, Phys. Rev. B 70, 115301 (2004).

[51] P. Comaron, G. Dagvadorj, A. Zamora, I. Carusotto, N. P. Proukakis, and M. H. Szymańska, Phys. Rev. Lett. 121, 095302 (2018). 